\title{
De Quervain's (subacute) thyroiditis onset after motor vehicle accident
}

\author{
Authors: Marin Glavčić ${ }^{1}$, Barbara Goršeta ${ }^{1}$, Nataša Buljan² (mentor) \\ 1 School of Medicine, University of Zagreb, Zagreb, Croatia \\ ${ }^{2}$ Department of Family Medicine, Andrija Štampar School of Public Health, School of Medicine \\ University of Zagreb, Zagreb, Croatia
}

DOI: https://doi.org/10.26800/LV-142-supp5-22

\section{Background:}

De Quervain's (subacute) thyroiditis is a painful swelling of the thyroid gland thought to be triggered by a viral infection. This condition is preceded in most cases by an acute respiratory infection.

\section{Case presentation:}

We report a case of a 40-year-old female who was presented with a fever, thyroid tenderness, enlarged lymph nodes, headache, weakness and pain in the upper jaw 8 weeks after motor vehicle accident. Patient didn't have any infections in the upper respiratory tract in the recent history and was tested SARS-CoV-2 negative at the time. Ultrasound showed an enlarged thyroid gland, especially the right lobe measuring $32 \times 28 \times 52 \mathrm{~mm}$ while the left lobe measured $27 \times 25 \times 54 \mathrm{~mm}$, the finding of which indicated subacute thyroiditis. Laboratory findings showed decreased TSH value and increased T3 and T4 values. Anti-Tg and Anti-TPO values were within the reference range. She was treated with NSAIDs, low-dose steroids and became asymptomatic after 6 weeks. The patient was prescribed a beta blocker due to occasional heart palpitations and excessive sweating due to thyrotoxicosis. Subsequent thyroid hormones and TSH controls showed a return to the reference values. Diffuse changes corresponding to the condition after subacute thyroiditis were still seen on ultrasound.

\section{Conclusion:}

Subacute thyroiditis can be triggered by stressful events from the recent past. Early recognition and early anti-inflammatory therapy will help in successful management of subacute thyroiditis. Therefore subacute thyroiditis should be considered in the diagnostic workup of fever, toxic symptoms of thyroid hormone excess, enlarged lymph nodes or headache several weeks after stressful event.

Keywords: Hyperthyroidism, Subacute, Thyroid Hormones, Thyroiditis 